EPJ Web of Conferences 103, 01007 (2015)

DOI: $10.1051 /$ epjconf/ 201510301007

(C) Owned by the authors, published by EDP Sciences, 2015

\title{
Laser Cooling of Lanthanides: from Optical Clocks to Quantum Simulators
}

\author{
A. Golovizin ${ }^{1,2}$, E. Kalganova $a^{1,2,3}$, G. Vishnyakova ${ }^{1,2,3}$, D. Tregubov ${ }^{1,2}, K_{\text {K. Khabarova }}^{1,2}$, \\ V. Sorokin ${ }^{1,2,3}$, and N. Kolachevsky ${ }^{1,2 a}$ \\ ${ }^{1}$ P.N. Lebedev Physical Institute, Leninsky prospect 53, 119991 Moscow, Russia \\ ${ }^{2}$ Moscow Institute of Physics and Technology, 141700 Dolgoprudny, Russia \\ ${ }^{3}$ Russian Quantum Center, Skolkovo, Moscow Region, Russia
}

\begin{abstract}
We discuss current progress in laser cooling of lanthanides (Er, Yb, Dy, Tm etc.) focusing on applications. We describe some important peculiarities taking Thulium atom as an example: Two stage laser cooling, trapping in an optical lattice, anisotropic interactions and spectroscopy of narrow transitions. Specific level structure and presence of magic wavelengths make ultracold Thulium a favorable candidate for optical clock applications. On the other hand, abundance of Feshbach resonances allow to tune interactions in ultracold gases and thus reach quantum degeneracy. It opens intriguing perspectives for novel quantum simulators employing dipole-dipole interactions in an optical lattice..
\end{abstract}

Keywords: lanthanides, laser cooling, optical clock, quantum degeneracy

One of the promptly developing areas in the broad field of laser cooling is the study of lanthanides. Significant progress was achieved in the past decade: such elements as Er [1], Dy[2], Ho [3] and Tm[4] were laser cooled and quantum degeneracy of Er and Dy was demonstrated. One of the unique features of these hollow $4 f$-subshell elements is the presence of the large ground state fine splitting corresponding to different $4 f$ electronic spin states. Also, due to large orbital moment of the hollow inner shell electrons $(L=3)$, most of these atoms possess large ground state magnetic moment up to $10 \mathrm{Bohr}$ magnetons. Combination of these features makes lanthanides particularly interesting for spectroscopy, study of ultra cold collisions and quantum degeneracy as well as for optical clock applications.

Despite complex level structure, laser cooling of lanthanides is surprisingly efficient. Typically, the laser cooling is realized in two stages: The first employs strong non-cycling transition in the blue spectral range, while narrow line cooling during the second stage allows cooling to $\mu \mathrm{K}$ range. Further cooling can be achieved by evaporation in an optical dipole trap which allows to reach quantum degeneracy. Meanwhile, study of Feshbach resonances in ultracold ensembles of lanthanides opens new exciting direction with growing number of publications (see e.g. [5]).

In our laboratory at Lebedev Institute we work on development of optical clock based on Tm [6]. The magnetic-dipole transition between ground state $\left(4 f^{3} 6 s^{2}\right)$ multiplet levels $J=7 / 2 \rightarrow J=5 / 2$ at 1.14 $\mu \mathrm{m}$ is suggested as a clock transition. For precision spectroscopy of this transition atoms should be cooled to $\mu \mathrm{K}$ range and loaded in an optical lattice.

The first stage laser cooling of Tm was demonstrated on strong transition at $410.6 \mathrm{~nm}$. Up to $10^{6}$

\footnotetext{
${ }^{a}$ Corresponding author: kolachevsky@lebedev.ru
} 
atoms were loaded in a magneto-optical trap (MOT) with the typical sub-Doppler temperature of 100 $\mu \mathrm{K}$. Efficient sub-Doppler cooling takes place directly in the MOT because of equal Landè g-factros of cooling levels. To reach lower temperatures, the second-stage cooling using the weak $530.7 \mathrm{~nm}$ transition was implemented. The transfer efficiency from the first to the second stage MOT approaches $100 \%$. Narrow line laser was used for the second stage cooling which allowed to reach temperatures of $10 \mu \mathrm{K}$. We observed reach physics of laser cooled Thulium. As an example, for particular detunings from the resonance one can observe s momentum-space crystals [7] as shown in Fig.1.

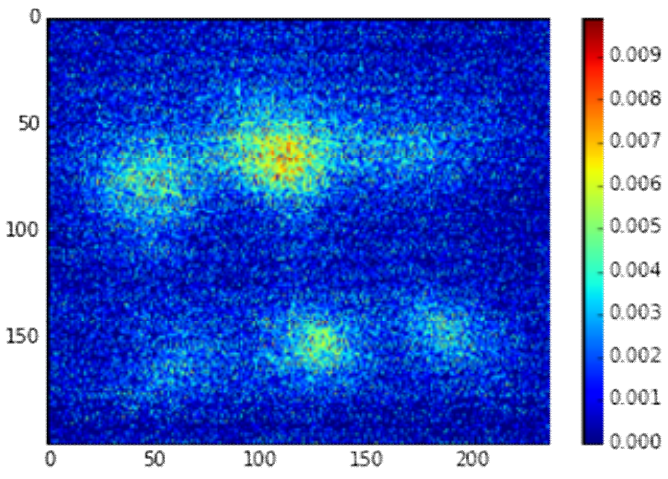

Figure 1. Momentum-space crystal in Tm MOT appearing at blue detuning of the second-stage cooling laser. Side image of 3D time-of-flight structure with cubic symmetry.

For further study Tm atoms were loaded in optical dipole trap. Currently we use $8 \mathrm{~W}$ of $530 \mathrm{~nm}$ laser radiation tightly focused in the MOT which allows to trap about $50 \%$ of atoms typical life time in a trap of $1 \mathrm{~s}$. Applying external magnetic field we studied Feshbach resonances in unpolarized Tm atoms at $10 \mu \mathrm{K}$. We detected one resonance at low magnetic field $(0.3 \mathrm{G})$ and plan to continue the study at lower temperatures. Investigation of Feshbach resonances gives important information about the scattering length which is crucial for quantum degeneracy.

Using a $1.14 \mu \mathrm{m}$ laser referenced to an ultra-stable ULE cavity we excited the clock transition in an optical lattice. We observe the spectral line width of $0.5 \mathrm{MHz}$ which significantly exceeds the natural line width $(1 \mathrm{~Hz})$. This undesired broadening results from inhomogeneous power broadening of the lower and upper clock levels which have significantly different dynamic polarizabilities at 530 $\mathrm{nm}$. For precision spectroscopy one needs to trap atoms in a lattice with the "magic" wavelength which, according to our calculations, should be around $800 \mathrm{~nm}$. We plan to set up the new lattice in the near future and continue study.

Lanthanides with large magnetic moments loaded in the 2D and 3D optical lattices are promising objects to study interactions in large quantum ensembles ("quantum simulators"). Since interaction Hamiltonian between atoms can be tuned by external magnetic field, one can efficiently simulate and study different types of crystals, superconductors and search for phase transitions.

\section{References}

1. A. J. Berglund, S. A. Lee, J. J. McClelland, Phys. Rev. A., 76, 053418 (2007)

2. S. H. Youn, M. Lu, U. Ray, B. L. Lev, Phys. Rev. A, 82, 043425 (2010)

3. B. Hemmerling, G. K. Drayna, E. Chae, A. Ravi, J. M. Doyle, New J. Phys. 16063070 (2014)

4. D. Sukachev, A. Sokolov, A Chebakov, A. Akimov, N. Kolachevsky, V. Sorokin, Phys. Rev. A., 82, 011405 (2010)

5. K. Baumann, N. Q. Burdick, M. Lu, B. L. Lev, Phys. Rev. A, 89, 020701(R) (2014)

6. N.N. Kolachevsky, Usp. Phys. Nauk 54, 863-870 (2011).

7. T. H. Loftus, T., Ido, M. M. , Boyd, A. D. , Ludlow, J. Ye, Phys. Rev. A, 70, 063413 . (2004)

8. A. Mecke, I. Lee, J.R. Baker jr., M.M. Banaszak Holl, B.G. Orr, Eur. Phys. J. E 14, 7 (2004)

9. M. Ben Rabha, M.F. Boujmil, M. Saadoun, B. Bessaïs, Eur. Phys. J. Appl. Phys. (to be published)

10. F. De Lillo, F. Cecconi, G. Lacorata, A. Vulpiani, EPL, 84 (2008)

11. L. T. De Luca, Propulsion physics (EDP Sciences, Les Ulis, 2009) 